\title{
Pirfenidone sensitizes NSCLC cells to the antitumor effect of Vinorelbine
}

\author{
C. Antunes $^{1,2,3}$, C.P.R. Xavier ${ }^{1,2}$, J. Oliveira ${ }^{4}$, L.L. Santos ${ }^{5}$, M.H. Vasconcelos ${ }^{1,2,6}$
}

1 i3S - Instituto de Investigação e Inovação em Saúde, University of Porto, Porto, Portugal; 2 Cancer Drug Resitance Group -IPATIMUP-Instituto de Patologia e Imunologia Molecular da Universidade do Porto, Porto, Portugal; 3 FMUP-Faculty of Medicine of the University of Porto, Porto, Portugal; 4 Department of Medical Oncology, IPO Portuguese Institute of Oncology, Porto, Portugal; 5 Experimental Pathology and Therapeutics Group and Surgical Oncology Department, IPO - Portuguese Institute of Oncology, Porto, Portugal; 6 Department of Biological Sciences, FFUP - Faculty of Pharmacy of the University of Porto, Porto, Portugal;

\section{Introduction}

Drug repurposing in cancer emerged as a strategy to identify antitumor potential in drugs clinically approved for the treatment of other diseases, offering new treatment possibilities especially for cancers with limited therapeutic alternatives [1], as the non-small cell lung cancer (NSCLC). Currently, Vinorelbine, an anti-mitotic drug, is used for the treatment of advanced NSCLC; however, it is often associated with high toxicity levels [2]. Hence, the combination of Vinorelbine with repurposed drugs would allow to reduce its concentration and thus the associated toxicity. Pirfenidone, an approved anti-fibrotic drug, appears as a possible repurposing contestant as it has been previously demonstrated to sensitize different cancer cells to the effect of some anti-cancer agents [3].

\section{Aim}

This study aimed to assess the potential off-target effect of Pirfenidone as a chemosensitizer of NSCLC cells to Vinorelbine, in order to reduce Vinorelbine's concentration and toxicity.

\section{Results}

Table 1 - The GI50 values of $\mathrm{NCl}-\mathrm{H} 460$ cell line treated with Vinorelbine and Pirfenidone, determined with the SRB assay.
Results are presented as mean $\pm \mathrm{SEM}$ of 3 independent experiments.
\begin{tabular}{|c|c|c|}
\hline Cancer Cell Line & $\mathbf{G I}_{\mathbf{5 0}}$ (nM) Vinorelbine at $\mathbf{4 8 h}$ & $\mathbf{G l}_{\mathbf{5 0}}$ (mM) Pirfenidone at $48 \mathrm{~h}$ \\
\hline $\mathrm{NCl}-\mathrm{H} 460$ & $6.9 \pm 0.46$ & $1.94 \pm 0.03$ \\
\hline
\end{tabular}

(B)

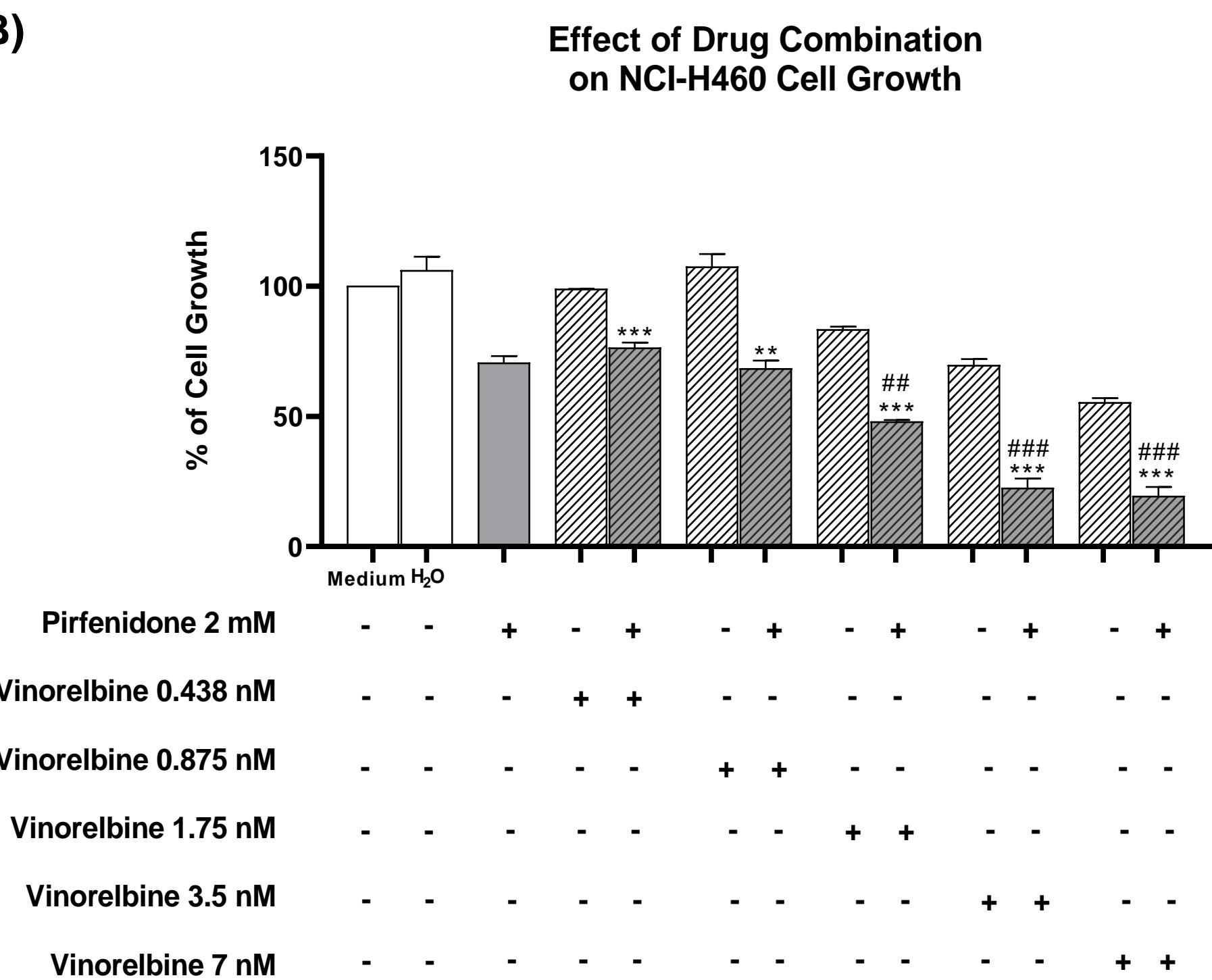

Figure 1 - Effect of the combination treatment of Vinorelbine and Pirfenidone in NCI-H460 cells. Determination of the \% of Figure 1 - Effect of the combination treatment of Vinorelbine and Pirfenidone in NCI-H460 cells. Determination of the \% of
cell growth of the sensitive NCI-H460 cell line, treated with drug combinations consisting of (A) Vinorelbine at $7 \mathrm{nM}$ with different concentrations of Pirfenidone; (B) Pirfenidone at $2 \mathrm{mM}$ with decreasing concentrations of Vinorelbine, using the SRB assay. Data represents the mean $\pm \operatorname{SEM}$ of 3 independent experiments. ${ }^{*} p<0.05,{ }^{* *}$ or $\# \# p<0.01,{ }^{* * *}$ or $\# \# p<0.001$, when comparing the effect of the drug combination with Vinorelbine (*) or Pirfenidone (\#) on their own.

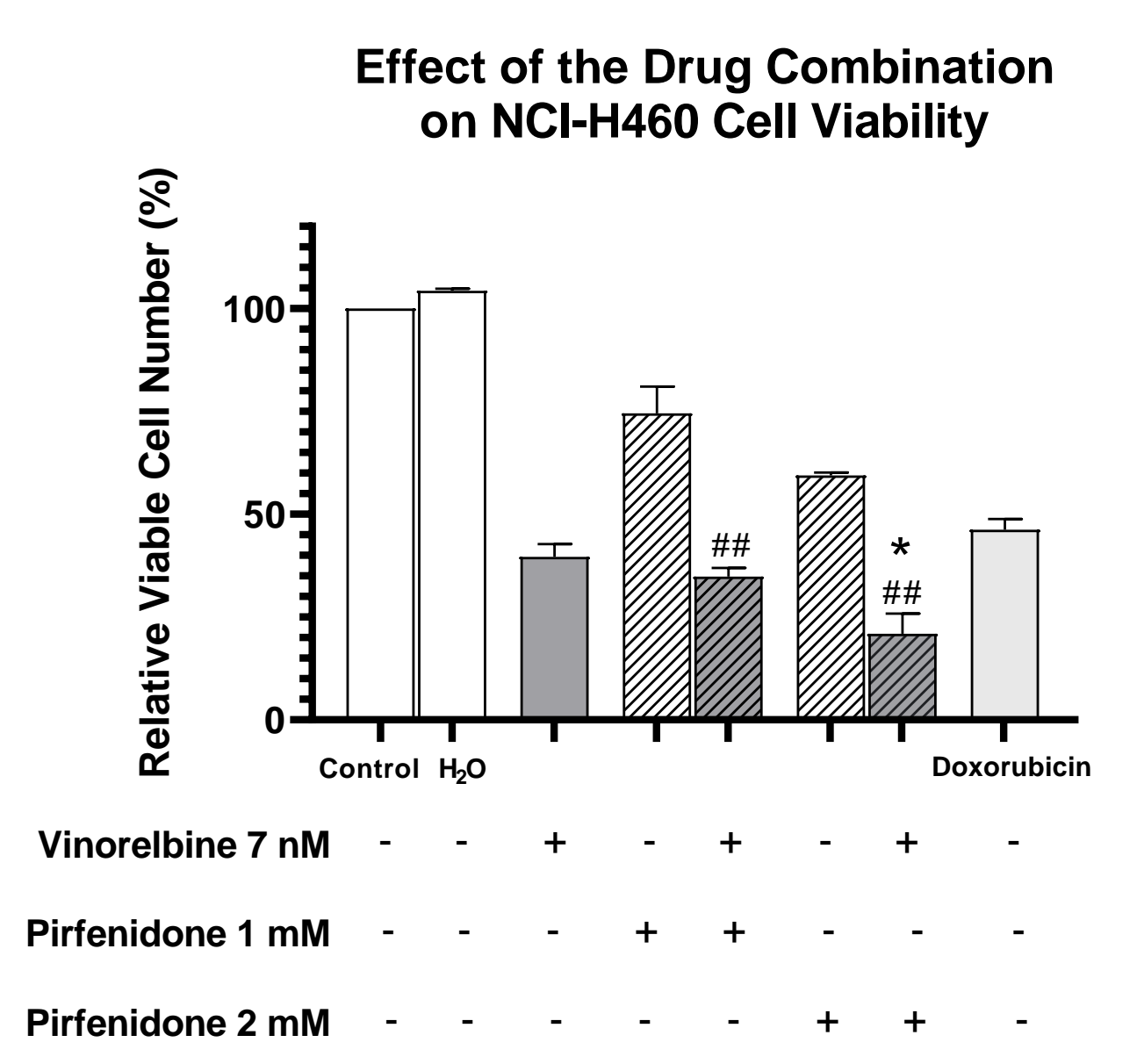

Figure 2 - Viable cell number of $\mathrm{NCl}-\mathrm{H} 460$ cells determined using the trypan blue exclusion assay. Cells were incubated with Vinorelbine and/or Pirfenidone for $48 \mathrm{~h}$. Doxorubicin was used as positive control. Data are expressed as mean \pm SEM from 3 independent experiments $(n=3) *{ }^{*}<0.05$; \# $p<0.01$, when comparing the effect of the drug combinations with Vinorelbin ${ }^{*}$ ) or Pirfenidone (\#) on their own.
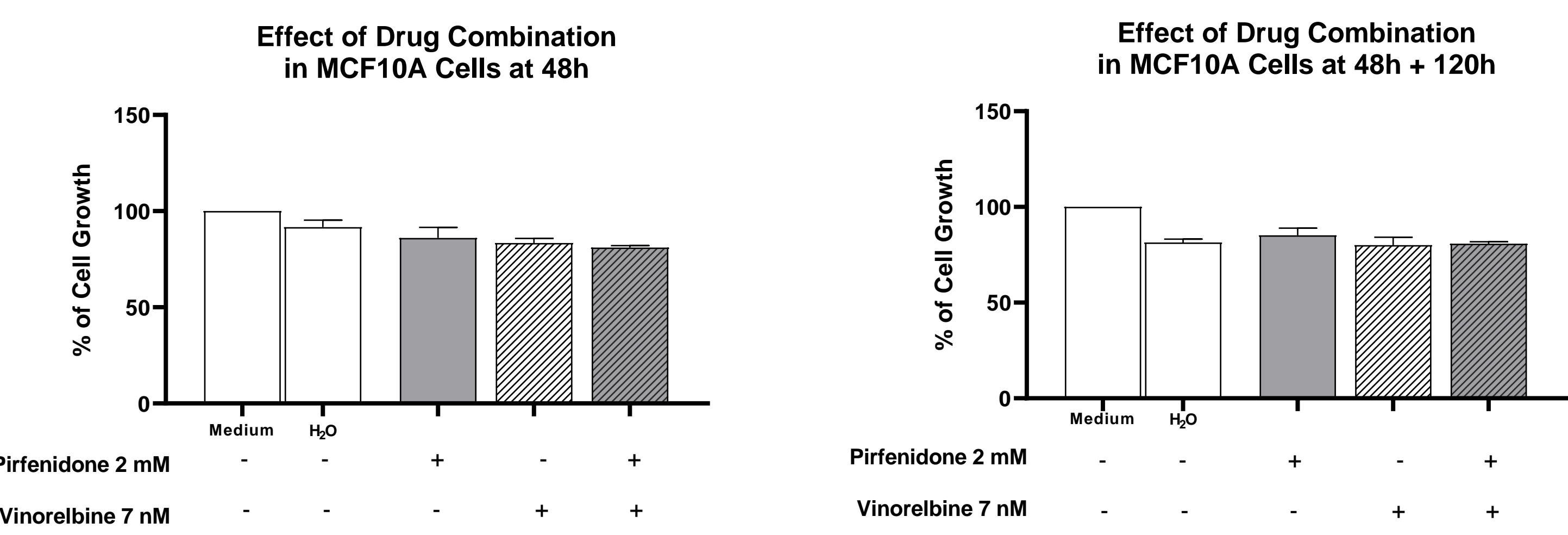

Figure 3 - Effect of the drug combination on non-tumorigenic cells. Determination of the \% of cell growth of the MCF $10 A$ non-tumorigenic breast cell line treated with the drug combination consisting of Vinorelbine at $7 \mathrm{nM}$ and Pirfenidone at 2 $\mathrm{mM}$, after (A) $48 \mathrm{~h}$ treatment or (B) $48 \mathrm{~h}$ treatment plus an additional $120 \mathrm{~h}$ without drug treatment, using the SRB assay. Results represent mean \pm SEM from 3 independent experiments $(n=3)$.

\section{References}

Verbaanderd, C., et al., Repurposing Drugs in Oncology: Next Steps. Trends Cancer, 2017. 3(8): p. 543-546

.

3. Mediavilla-Varela, M., et al., The anti-fibrotic agent pirfenidone synergizes with cisplatin in killing tumor cells and cancer-associated fibroblasts. BMC Cancer, 2016. 16: p. 176.

\section{Methodologies}

- Sulforhodamine B Assay;

- Trypan Blue Exclusion Assay;

- Cell Cycle Profile analysis by Flow Cytometry;

- BrdU Incorporation Assay.

(A)
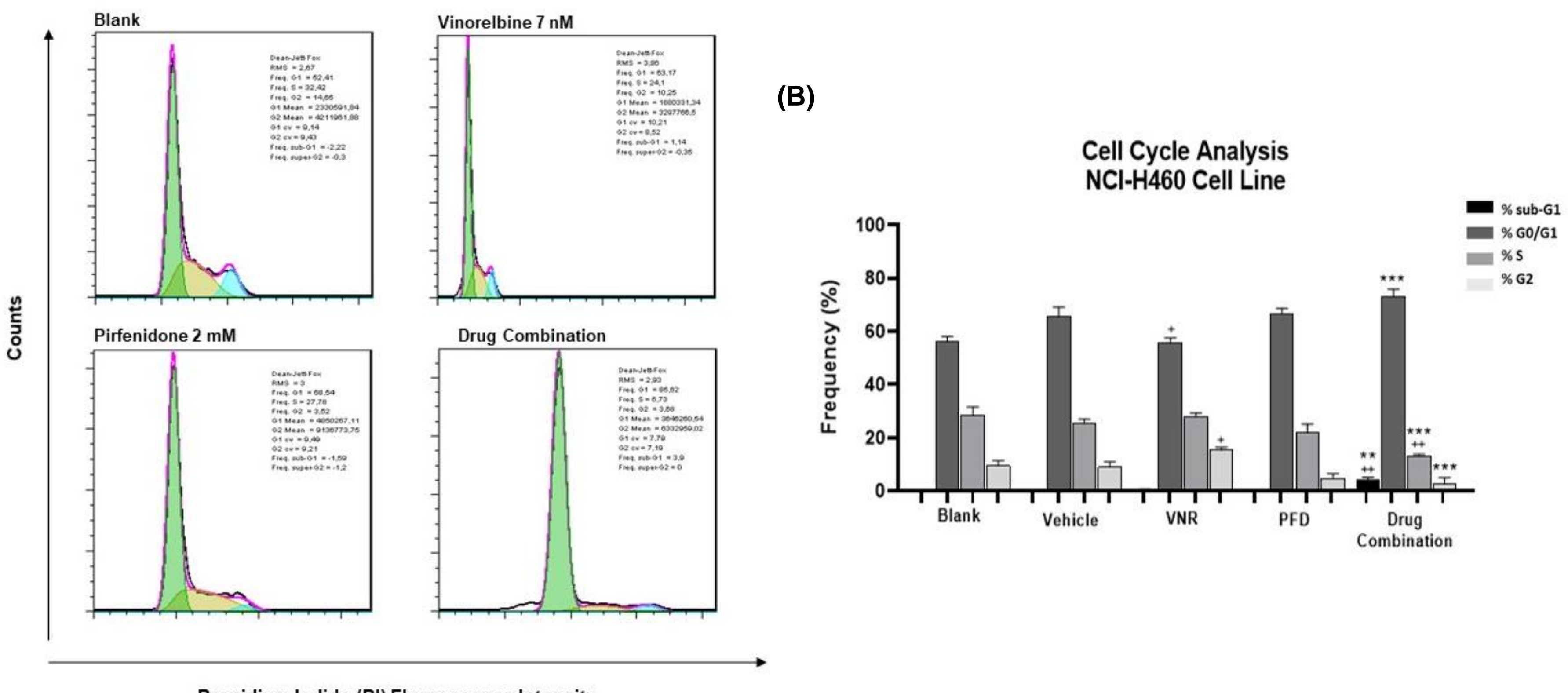

Figure 4 - Effect of the drug combination on the cell cycle profile of $\mathrm{NCl}-\mathrm{H} 460$ cells. Cells were treated with Vinorelbine at $\mathrm{nM}$ and/or Pirfenidone at $2 \mathrm{mM}$, and the cell cycle profile was analysed by flow cytometry. (A) Representative cell cycle 3 independent experiments $+p<0.05 ; * *$ or. $+p<<0.01 ; * * * p<0.001$, when comparing the effect of the treatment condition with Vinorelbine $(*)$ or the vehicle $(+)$
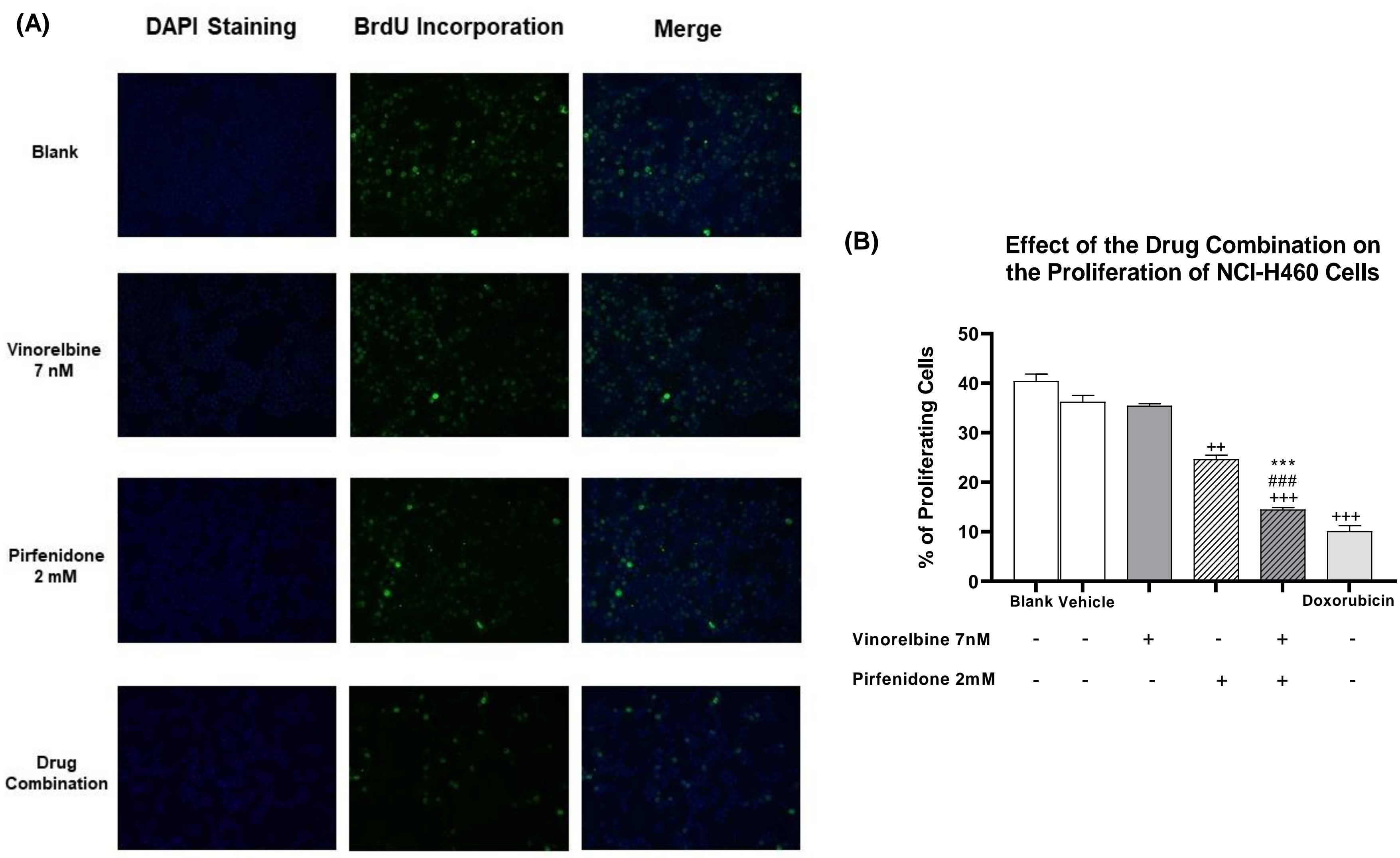

Figure 5 - Effect of the drug combination on NCI-H460 cell proliferation. (A) Representative fluorescence microscopy images (20X amplification) of the BrdU incorporation assay: DAPI staining represents the total cell population (blue stained nuclei); BrdU Incorporation represents proliferating cells (green); (B) \% of Proliferating Cells. Results represent mean \pm SEM of 3 independent experiments. Doxorubicin was used as a positive control. $++\mathrm{p}<0.01 ; * * *$ \#\#\# or $+++\mathrm{p}<0.001$, when .

\section{Conclusion}

Results show that the drug combination decreases NSCLC cell growth and cell viability, promoting cell cycle arrest and reducing cellular proliferation. Future in vitro and in vivo studies will validate these results, to further evaluate the possibility of repurposing Pirfenidone for the adjuvant treatment of NSCLC in combination with Vinorelbine. 